Ferrata Storti Foundation

\title{
Haploidentical versus unrelated allogeneic stem cell transplantation for relapsed/ refractory acute myeloid leukemia: a report on 1578 patients from the Acute Leukemia Working Party of the EBMT
}

Haematologica 2019

Volume 104(3):524-532

\section{Correspondence:}

EOLIA BRISSOT

eolia.brissot@aphp.fr

Received: February 4, 2018.

Accepted: October 19, 2018.

Pre-published: October 25, 2018.

doi:10.3324/haematol.2017.187450

Check the online version for the most updated information on this article, online supplements, and information on authorship \& disclosures: www. haematologica.org/content/104/3/524

\section{(C)2019 Ferrata Storti Foundation}

Material published in Haematologica is covered by copyright. All rights are reserved to the Ferrata Storti Foundation. Use of published material is allowed under the following terms and conditions:

https://creativecommons.org/licenses/by-nc/4.0/legalcode. Copies of published material are allowed for personal or internal use. Sharing published material for non-commercial purposes is subject to the following conditions:

https://creativecommons.org/licenses/by-nc/4.0/legalcode, sect. 3. Reproducing and sharing published material for commercial purposes is not allowed without permission in writing from the publisher.

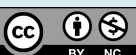

\section{Eolia Brissot, ${ }^{1}$ Myriam Labopin, ${ }^{1,2}$ Gerhard Ehninger, ${ }^{3}$ Matthias Stelljes, ${ }^{4}$ Arne Brecht, ${ }^{5}$ Arnold Ganser, ${ }^{6}$ Johanna Tischer, ${ }^{7}$ Nicolaus Kröger, ${ }^{8}$ Boris Afanasyev, ${ }^{9}$ Jürgen Finke, ${ }^{10}$ Ahmet Elmaagacli, ${ }^{11}$ Herman Einsele, ${ }^{12}$ Mohamad Mohty $^{1,2 *}$ and Arnon Nagler ${ }^{2,13 *}$}

\begin{abstract}
${ }^{1}$ Service d'Hématologie Clinique et de Thérapie Cellulaire, Hôpital Saint Antoine, APHP, Paris, France; ${ }^{2}$ Acute Leukemia Working Party office, Hôpital Saint Antoine, APHP, Paris, France; ${ }^{3}$ Universitaetsklinikum Dresden, Medizinische Klinik und Poliklinik I, Germany; ${ }^{4}$ University of Münster, Department of Medicine A / Hematology and Oncology, Germany; ${ }^{5}$ Deutsche Klinik für Diagnostik, KMT Zentrum, Wiesbaden, Germany; ${ }^{6} \mathrm{Hannover} \mathrm{Medical}$ School, Department of Haematology, Hemostasis, Oncology, and Stem Cell Transplantation, Germany; ' Klinikum Grosshadern, Med. Klinik III, Munich, Germany; ${ }^{8}$ University Hospital Eppendorf, Bone Marrow Transplantation Centre, Hamburg, Germany; ${ }^{9}$ First State Pavlov Medical University of St. Petersburg, Raisa Gorbacheva Memorial Research Institute for Pediatric Oncology, Hematology, and Transplantation, Russia; ${ }^{10}$ University of Freiburg, Faculty of Medicine and Department of Medicine -Hematology, Oncology and Stem Cell Transplantation, Germany; ${ }^{11}$ Asklepios Klinik St. Georg, Department of Hematology, Hamburg, Germany; ${ }^{12}$ Universitaetsklinikum Würzburg, Med. Klinik und Poliklinik II, Germany and ${ }^{13} \mathrm{Hematology}$ Division, Chaim Sheba Medical Center and Tel Aviv University, Tel-Hashomer, Ramat-Gan, Israel
\end{abstract}

* MM and AN contributed equally to this study and share senior authorship

\section{ABSTRACT}

P rimary refractory or relapsed acute myeloid leukemia is associated with a dismal prognosis. Allogeneic stem cell transplantation is the only therapeutic option that offers prolonged survival and cure in this setting. In the absence of a matched sibling donor, transplantation from unrelated 10/10 HLA allele-matched or 9/10 HLA allele-mismatched donors and haploidentical donors are potential alternatives. The current study aimed to compare the outcomes of acute myeloid leukemia patients with active disease who received allogeneic stem cell transplantation from a haploidentical donor with post-transplant cyclophosphamide $(\mathrm{n}=199)$ versus an unrelated 10/10-matched donor $(n=1111)$ and versus an unrelated 9/10-mismatched donor ( $\mathrm{n}=383$ ) between 2007 and 2014 and who were reported to the European Society for Blood and Marrow Transplantation registry. Propensity score weighted analysis was conducted in order to control for disease risk imbalances between the groups. The leukemia-free survival rates at 2 years of recipients of grafts from a haploidentical donor, an unrelated 10/10-matched donor and an unrelated 9/10-mismatched donor were $22.8 \%, 28 \%$ and $22.2 \%$, respectively $(P=\mathrm{NS})$. In multivariate analysis, there were no significant differences in leukemia-free survival, overall survival, relapse incidence, non-relapse mortality, or graft-versus-host-disease-free relapse-free survival between the three groups. Two predictive factors were associated with a higher relapse incidence: transplantation during first or second relapse compared to primary refractory acute myeloid leukemia and poor cytogenetics. Allogeneic stem cell transplantation may rescue about $25 \%$ of acute myeloid leukemia patients with active disease. Importantly, the outcomes of transplants from haploidentical donors were comparable to those from 10/10-matched and 9/10-mismatched unrelated donors. Therefore, a haploidentical donor is a valid option for acute myeloid leukemia patients with active disease. 


\section{Introduction}

After initiation of intensive chemotherapy for acute myelogenous leukemia (AML), failure to respond is a major unfavorable prognostic factor. ${ }^{1,2}$ Obtaining a morphological complete remission (CR) after induction has been defined as a prognostic factor and even, until recently, considered as a prerequisite for allogeneic stem cell transplantation (HSCT). However, up to $30 \%$ of adults with newly diagnosed AML fail to achieve CR after two courses of intensive chemotherapy. ${ }^{1}$ Moreover, once a first or second CR has been obtained, approximately half of younger patients and $80 \%$ of older patients relapse. ${ }^{1,3,4}$ In both clinical situations, refractory and/or relapsed AML, active disease remains a major therapeutic challenge. Consequently, the accumulating evidence that HSCT can deliver long-term disease-free survival in a proportion of patients with AML with active disease represents an importance advance in the treatment in this very high-risk patient population. ${ }^{5-}$ ${ }^{9}$ Defining the impact of donor selection is still a major issue. It has been demonstrated that HSCT from matched sibling donors is a valid option, leading to a disease-free survival rate in the range of $20-30 \%$ for this very high-risk patient population.,10-13 More recently, HSCT from unrelated donors (UD) was used for patients with primary refractory AML, with an overall survival (OS) rate of about $22 \% \cdot{ }^{13-15}$ Since 2010 , the use of haploidentical HSCT has surged by about 300\% among European Society of Blood and Marrow Transplantation (EBMT) centers. ${ }^{16,17}$ Indeed, over recent years, haploidentical donors have been increasingly adopted as a valid source of donor cells for HSCT in AML in the absence of HLA-compatible matched sibling donors or matched UD. Based on several non-randomized comparative studies evaluating HSCT from haploidentical donors (Haplo HSCT), ${ }^{18-21}$ the combined data suggest similar outcomes for Haplo and UD HSCT. ${ }^{22,23}$ However, only small series are available for patients with resistant and/or relapsed AML undergoing HSCT from alternative donors. Craddock et al. reported that the OS rate of 36 patients who received an UD transplant with a reduced-intensity conditioning regimen (RIC) was 36\% at 5 years, which was similar to that of 18 patients given myeloablative conditioning (MAC). ${ }^{14}$ For patients with active leukemia, HSCT from alternative or mismatched donors may, theoretically, be of advantage, as HLA disparities may augment donor/recipient alloreactivity.

However, relatively few data are available for the very high-risk population of patients with refractory or relapsing AML transplanted from alternative donors while in active disease. In view of the fact that the development of Haplo HSCT is significantly influenced by the use of post-transplantation cyclophosphamide (PTCy), and because advances in supportive care influence outcomes, a safety and efficacy update comparison between Haplo and UD HSCT in a large cohort of patients with active disease is highly warranted to further support decision-making. With this aim, the present study, based on the EBMT - Acute Leukemia Working Party (ALWP) database, was conducted in order to compare outcomes of AML patients with active disease after Haplo HSCT versus $10 / 10$ or $9 / 10$ HLA-matched UD HSCT.

\section{Methods}

\section{Study design and data retrieval}

This is a retrospective, multicenter, registry-based analysis. Data for this study were provided and approved by the ALWP of the EBMT group registry. The EBMT registry is a voluntary working group of more than 600 transplant centers, mostly located in Europe, which are required to report all consecutive stem-cell transplantations and follow-up data once a year. Data are entered, managed, and maintained in a central database with internet access; each EBMT center is represented in this database. There are no restrictions on centers for reporting data, except for those required by law on patients' consent, data confidentiality and accuracy. Quality control measures include several independent systems: confirmation of the validity of the entered data by the reporting team, selective comparison of the survey data with MED-A data sets in the EBMT registry database, cross-checking with national registries, and regular in-house and external data audits. All patients provided informed consent to the use of their data in retrospective studies. The Review Board of the ALWP as well as the ethics committee of the EBMT approved this study.

Eligibility criteria for this analysis included adult patients (aged $>18$ years) with active AML including primary refractory AML, AML in first relapse and second relapse who had received a first HSCT from a 10/10 HLA allele-matched UD (UD 10/10), or a 9/10 HLA allele-mismatched UD (UD 9/10) or a haploidentical donor ( $\geq 2$ antigen mismatches or more out of 8 ) with post-transplant cyclophosphamide (Haplo PTCy) as graft-versus-host disease (GvHD) prophylaxis. Active AML was defined by the failure to achieve CR (bone marrow blasts $>5 \%$ ) despite induction chemotherapy. Cytogenetic groups were defined according to Grimwade et al. ${ }^{24}$ The source of stem cells could be either bone marrow or granulocyte colony-stimulating factor-mobilized peripheral blood stem cells. All UD were HLA-matched (10/10) or mismatched at one loci $(9 / 10)$ (-A, -B, -C, -DRB1, -DOB1). We excluded patients who had undergone umbilical cord blood HSCT, so that the analysis was restricted to a more homogeneous study population. MAC was defined, according to the EBMT, as a regimen containing total body irradiation with a dose $>6 \mathrm{~Gy}$, a total dose of oral busulfan $>8 \mathrm{mg} / \mathrm{kg}$ or a total dose of intravenous busulfan $>6.4 \mathrm{mg} / \mathrm{kg}{ }^{25}$ The FLAMSA sequential conditioning regimen consisted of a combination of a short course of intensive chemotherapy using fludarabine $30 \mathrm{mg} / \mathrm{m}^{2} /$ day, intermediate-dose cytosine arabinoside $2 \mathrm{~g} / \mathrm{m}^{2} /$ day, and amsacrine $100 \mathrm{mg} / \mathrm{m}^{2} /$ day from day -12 to -9 , followed, after a 3-day rest, by RIC using $4 \mathrm{~Gy}$ total body irradiation on day -5 , cyclophosphamide 40 to 60 $\mathrm{mg} / \mathrm{kg} /$ day on days -4 and -3 , and antithymocyte globulin from days -4 to -2 ; the 4 Gy total body irradiation could have been replaced by a total dose of intravenous busulfan of $6.4 \mathrm{mg} / \mathrm{kg}$ (or an equivalent oral dose). ${ }^{26-28}$

\section{Endpoints}

OS was calculated from the date of transplantation until death or last observation alive. Leukemia-free survival (LFS) was calculated from the date of transplantation until relapse or last diseasefree follow-up. Relapse and death from any cause were considered events. Non-relapse mortality (NRM) was defined as death without prior relapse. Neutrophil recovery was defined as achieving absolute neutrophil counts greater than or equal to $0.5 \times 10^{9} / \mathrm{L}$ for three consecutive days. The diagnosis and grading of acute ${ }^{29}$ and chronic $\mathrm{GvHD}^{30}$ were performed by transplant centers using the standard criteria. Cytogenetic abnormalities were classified according to Medical Research Centre criteria, and graft-versushost-free, relapse-free survival (GRFS) as previously published. ${ }^{31}$ 


\section{Statistical analysis}

Patient-, disease-, and transplant-related variables were compared between the three groups (Haplo PTCy, UD 10/10, UD 9/10) using the chi-square test for categorical variables and the Mann-Whitney test for continuous variables. The median followup was estimated using the reverse Kaplan-Meier method. Variables considered were patient's age at transplantation, donor/recipient gender, interval from diagnosis to transplantation, cytogenetic group, type of conditioning (RIC/MAC/FLAMSA), source of stem cells (peripheral blood stem cells versus bone marrow), patient/donor cytomegalovirus serology, Karnofsky Performance Status (KPS) at the time of transplantation, in vivo Tcell depletion, and year of transplantation. Factors that significantly differed between the three groups with $P$-values of $<0.05$, and all known as potential prognostic factors were included in the final models. Cumulative incidence functions were used to estimate relapse incidence (RI) and NRM in a competing risk setting, because death and relapse compete with each other. To study chronic GvHD, we considered relapse and death to be competing events. Probabilities of LFS and OS were calculated using KaplanMeier estimates. Univariate analyses were performed using the Gray test for cumulative incidence functions and the log-rank test for LFS and OS. Associations of patient and graft characteristics with outcomes were evaluated in multivariate analysis, using a Cox proportional hazards model. All tests were two-sided.

We used propensity score weighing to control for pre-treatment imbalances in observed variables. The following factors were included in the propensity score model: patient's age, time from diagnosis to transplantation, year of transplant, status at transplant, cytogenetic group, donor/patient cytomegalovirus serology, conditioning (RIC versus MAC), and gender matching (female donor to male recipient versus other). Propensity scores were estimated using generalized boosted models.

As the study question was whether Haplo PTCy could replace UD 10/10 or UD 9/10, we weighted the groups receiving either UD $10 / 10$ or UD $9 / 10$ HSCT to match the characteristics of patients receiving Haplo HSCT, by estimating the average treatment effect among the treated group (Haplo HSCT being the treated group). We then used pairwise average treatments to fit the weighted Kaplan-Meier and Cox models separately for Haplo PTCy versus UD 10/10 HSCT and Haplo PTCy versus UD 9/10 HSCT.

The type I error rate was fixed at 0.05 for determination of factors associated with time to events. Analyses were performed using the R statistical software version 3.2.3 (R Development Core Team, Vienna, Austria). Propensity score analysis was performed using the mnps function of the Twang package and weighted analyses using the survey package.

\section{Results}

\section{Patients, disease and transplant characteristics}

Data were obtained from 218 reporting centers (Online Supplementary Data). The patients' and disease characteristics are summarized in Table 1. Of the total 1693 HSCT, 1111 were UD 10/10, 383 were UD 9/10 and 199 were Haplo PTCy. The three cohorts of patients differed for several variables (Table 1). The median follow-up was longer for the UD 10/10 and the UD 9/10 groups than for the Haplo-PTCy group. The follow-up completeness index at 2 years, which is the ratio of total observed person-time and the potential person-time of follow-up at 2 years $^{32}$ was $73 \%$ for Haplo PTCy, 76\% for UD 10/10 and $80 \%$ for UD $9 / 10$. Significantly more patients received
MAC regimens in the Haplo PTCy group in comparison to both the UD 10/10 and the UD 9/10 groups. There were more cytomegalovirus-positive recipient-donor pairs in the Haplo-PTCy group. Peripheral blood stem cells were, as expected, the main source of stem cells in the UD 10/10 and UD 9/10 groups, while peripheral blood stem cells represented $52.8 \%$ of the stem cell source in the HaploPTCy group.

\section{Engraftment and graft-versus-host disease}

The cumulative incidence of engraftment at day 30 was $85.5 \%$ [95\% confidence interval (95\% CI): 79-90.2], 92.3\% (95\% CI: 90.5-93.7) and 92.2\% (95\% CI: 88.9-94.6) in the Haplo PTCy, UD 10/10 and 9/10 groups, respectively $\left(\mathrm{P}<10^{-3}\right.$ for both comparisons).

Lower incidences of all acute GvHD grades were observed after Haplo PTCy than after UD 9/10. The cumulative incidences of grade II-IV acute GvHD were $28.2 \%$ and $36.3 \%$, respectively $(P=0.03)$ and those of severe grade III-IV acute GvHD were $8.9 \%$ and $16.1 \%$, respectively $(P=0.02)$ (Table 3$)$. No difference was observed in the incidence of grade II-IV acute GvHD between the Haplo PTCy and UD 10/10 groups ( $P=\mathrm{NS}$ ).

At 2 years, the cumulative incidence of chronic GvHD was lower in the Haplo PTCy group than in the UD 9/10 group $(19.3 \%$ and $27.4 \%$, respectively, $P=0.04)$, while no difference was found in the incidence of chronic GvHD between the Haplo PTCy and UD $10 / 10$ groups $(P=\mathrm{NS}$ ) (Table 3). The cumulative incidence of extensive chronic GvHD was similar in the three groups of patients (Haplo PTCy - 11\%, UD 10/10 -11.6\% and UD 9/10 -11.6\%).

The percentages of patients who achieved CR within 100 days were $79.7 \%, 77 \%$ and $78.3 \%$ in the Haplo PTCy, UD 10/10 and UD 9/10 groups, respectively $(P=N S)$. Performing a landmark analysis at day 100 for comparing outcomes between patients who achieved CR before day 100 to those who did not indicated that CR seems to be a surrogate marker for subsequent outcome. The probability of being alive and free of disease 1 year after HSCT was only $13.7 \%$ for patients who did not achieve CR before day 100 (data not shown).

We also analyzed chronic GvHD as a time-dependent variable demonstrating that the association of chronic GvHD with a lower RI hazard ratio $(\mathrm{HR})=0.77$, 95\% CI: 0.60-0.99, $P=0.04$ ) was counterbalanced by a higher NRM $\left(\mathrm{HR}=1.98\right.$, 95\% CI: 1.38-2.84, $\left.\mathrm{P}<10^{-3}\right)$, and thus did not translate into better LFS $(P=\mathrm{NS})$.

\section{Leukemia-free survival, overall survival, relapse incidence and non-relapse mortality}

In univariate analysis, the LFS rate at 2 years was $22.8 \%$ in the Haplo PTCy group versus $28 \%$ in the UD $10 / 10$ group and $22.2 \%$ in the UD $9 / 10$ group $(P=\mathrm{NS}$ ) (Figure 1A, Online Supplementary Table S1). Multivariate analysis showed lower LFS rates in patients with poor cytogenetics ( $\mathrm{HR}=1.35,95 \% \mathrm{CI}: 1.11-1.62, P=0.002)$, those transplanted in second relapse in comparison to those transplanted in primary refractory $\mathrm{AML}(\mathrm{HR}=1.31,95 \% \mathrm{CI}, 1.03-1.65$, $P=0.03)$, and in patients transplanted from cytomegalovirus seropositive donors $(\mathrm{HR}=1.22,95 \% \mathrm{CI}$ : 1.05-1.41, $P=0.01)$. In contrast, better LFS was associated with $\mathrm{KPS} \geq 90$ at transplantation (HR=0.66, 95\% CI: 0.58 $\left.0.76, P<10^{-3}\right)$, with a shorter time from diagnosis to transplantation ( $\mathrm{HR}=0.99$, 95\% CI: $0.98-0.99, P=0.02)$, and with RIC in comparison to MAC (HR=0.84, 95\% CI: 0.71 - 
$0.99, P=0.03)$. Of note, no effect was observed for donor type (Table 4).

The OS rate at 2 years did not differ between the three groups of patients $29.3 \%$ in the Haplo PTCy group versus $34.7 \%$ in the UD 10/10 group and $27.6 \%$ in the UD 9/10 group, $P=\mathrm{NS}$ ) (Figure 1B). These results were confirmed by multivariate analysis. In the latter, three predictive fac- tors were associated with lower OS: disease status (second relapse versus primary refractory $\mathrm{AML}$ ), poor cytogenetics and the patient being positive for cytomegalovirus, whereas KPS $\geq 90$ at transplant, RIC versus MAC and shorter time from diagnosis to transplantation were associated with a better OS (Table 4).

We did not find any differences in terms of RI between

Table 1. Baseline characteristics of patients.

\begin{tabular}{|c|c|c|c|c|c|}
\hline Number & $\begin{array}{c}\text { Haplo PTCy } \\
199\end{array}$ & $\begin{array}{c}\text { UD 10/10 } \\
1111\end{array}$ & $\begin{array}{l}\text { UD } 9 / 10 \\
383\end{array}$ & $\begin{array}{l}\text { Haplo versus } \\
\text { UD 10/10 } \\
\text { P value }\end{array}$ & $\begin{array}{l}\text { Haplo versus } \\
\text { UD } 9 / 10 \\
P \text { value }\end{array}$ \\
\hline Follow-up in months, median (range) & $16(2.1-63.5)$ & $18.1(0.6-113)$ & $22.9(1.8-104.9)$ & 0.05 & 0.02 \\
\hline Age at transplant in years, median (range) & $51.9(18.2-77.8)$ & $52.4(18.1-77.3)$ & $51.7(18-76)$ & NS & NS \\
\hline Year of transplant in years, median (range) & $2014(2009-2015)$ & $2011(2007-2015)$ & 2011(2007-2015) & $<10^{-3}$ & $<10^{-3}$ \\
\hline $\begin{array}{l}\text { Time from diagnosis in transplant in years } \\
\text { in months (range) }\end{array}$ & $7.6(2-122.1)$ & $6.8(2-474.8)$ & $8.1(2.1-121.4)$ & NS & NS \\
\hline $\begin{array}{l}\text { Status of AML, n (\%) } \\
\text { Primary refractory } \\
1^{\text {st }} \text { relapse } \\
2^{\text {nd }} \text { relapse }\end{array}$ & $\begin{array}{l}82(41.2) \\
87(43.7) \\
30(15.1)\end{array}$ & $\begin{array}{l}501(45.1) \\
491(44.2) \\
119(10.7)\end{array}$ & $\begin{array}{l}141(36.8) \\
190(49.6) \\
52(13.6)\end{array}$ & NS & NS \\
\hline $\begin{array}{l}\text { Cytogenetics, n (\%) } \\
\text { Favorable } \\
\text { Intermediate } \\
\text { Adverse } \\
\text { Unknown/failed }\end{array}$ & $\begin{array}{c}7(5.6) \\
83(65.9) \\
36(28.6) \\
73\end{array}$ & $\begin{array}{c}40(10.2) \\
235(59.8) \\
118(30) \\
718\end{array}$ & $\begin{array}{c}15(9.3) \\
98(60.9) \\
48(29.8) \\
222\end{array}$ & NS & NS \\
\hline $\begin{array}{l}\text { KPS at transplant, n (\%) } \\
<90 \% \\
\geq 90 \%\end{array}$ & $\begin{array}{l}91(49.7) \\
92(50.3)\end{array}$ & $\begin{array}{l}412(49) \\
619(61)\end{array}$ & $\begin{array}{l}143(39.7) \\
217(60.3)\end{array}$ & 0.01 & 0.03 \\
\hline $\begin{array}{l}\text { Patients' gender, n (\%) } \\
\text { Male } \\
\text { Female }\end{array}$ & $\begin{array}{c}108(54.3) \\
91(45.7)\end{array}$ & $\begin{array}{l}587(52.9) \\
523(47.1)\end{array}$ & $\begin{array}{l}209(54.6) \\
174(45.4)\end{array}$ & NS & NS \\
\hline $\begin{array}{l}\text { Donors' gender, n (\%) } \\
\text { Male } \\
\text { Female }\end{array}$ & $\begin{array}{l}119(59.8) \\
80(40.2)\end{array}$ & $\begin{array}{l}769(72.8) \\
287(27.2)\end{array}$ & $\begin{array}{l}247(66) \\
127(34)\end{array}$ & $<10^{-3}$ & NS \\
\hline $\begin{array}{l}\text { Female D to male R, n (\%) } \\
\text { No } \\
\text { Yes }\end{array}$ & $\begin{array}{c}155(77.9) \\
44(22.1)\end{array}$ & $\begin{array}{l}925(87.7) \\
130(12.3)\end{array}$ & $\begin{array}{c}313(83.7) \\
61(16.3)\end{array}$ & $<10^{-3}$ & NS \\
\hline $\begin{array}{l}\text { CMV status, n (\%) } \\
\text { D-/R- } \\
\text { D+/R- } \\
\text { D-/R+ } \\
\text { D+/R+ }\end{array}$ & $\begin{array}{c}30(15.7) \\
9(4.71) \\
39(20.4) \\
113(59.2)\end{array}$ & $\begin{array}{c}308(29.1) \\
81(7.6) \\
305(28.8) \\
366(34.5)\end{array}$ & $\begin{array}{l}89(24.2) \\
38(10.3) \\
128(34.9) \\
112(30.5)\end{array}$ & $<10^{-3}$ & $<10^{-3}$ \\
\hline $\begin{array}{l}\text { Conditioning regimen, n (\%) } \\
\text { Myeloablative } \\
\text { Reduced intensity } \\
\text { Sequential strategy }\end{array}$ & $\begin{array}{c}106(53.5) \\
81(40.9) \\
11(5.6)\end{array}$ & $\begin{array}{l}465(41.9) \\
380(34.3) \\
263(23.7)\end{array}$ & $\begin{array}{l}144(37.7) \\
128(33.5) \\
110(28.8)\end{array}$ & $<10^{-3}$ & $<10^{-3}$ \\
\hline $\begin{array}{l}\text { Source of stem cells, n (\%) } \\
\text { Bone marrow } \\
\text { Peripheral blood }\end{array}$ & $\begin{array}{l}94(47.2) \\
105(52.7)\end{array}$ & $\begin{array}{c}72(6.5) \\
1039(93.5)\end{array}$ & $\begin{array}{c}30(7.8) \\
353(92.2)\end{array}$ & $<10^{-3}$ & $<10^{-3}$ \\
\hline $\begin{array}{l}\text { GvHD prophylaxis, n (\%) } \\
\text { CSA+MTX } \\
\text { CSA+MMF } \\
\text { Tacrolimus+MMF } \\
\text { CSA+MMF+MTX } \\
\text { PTCy }\end{array}$ & $\begin{array}{c}0 \\
0 \\
0 \\
0 \\
199(100)\end{array}$ & $\begin{array}{c}325(30) \\
484(44.6) \\
54(5) \\
20(2) \\
25(2.3)\end{array}$ & $\begin{array}{c}113(29.7) \\
161(42.4) \\
27(7.1) \\
11(2.9) \\
7(2)\end{array}$ & $<10^{-3}$ & $<10^{-3}$ \\
\hline $\begin{array}{l}\text { In vivo T-cell depletion, } \mathrm{n}(\%) \\
\text { No } \\
\text { Yes }\end{array}$ & $\begin{array}{c}188(94.5) \\
11(5.5)\end{array}$ & $\begin{array}{l}265(24.2) \\
830(75.8)\end{array}$ & $\begin{array}{c}54(14.2) \\
327(85.8)\end{array}$ & $<10^{-3}$ & $<10^{-3}$ \\
\hline
\end{tabular}

AML: acute myeloid leukemia; BM: bone marrow; CsA: cyclosporine; D: donor; GvHD: graft-versus-host disease; Haplo: haplo-identical; KPS: Karnofsky Performance Status; MMF: mycophenolate mofetil; MTX: methotrexate; NS: not significant; PTCy: post-transplant cyclophosphamide; R: recipient; UD: unrelated donor. 
the three groups (Table 2, Figure 1C). This result was also confirmed in multivariate analysis showing that poor cytogenetics and disease status (first and second relapse versus primary refractory AML) were the only risk factors associated with increased RI, whereas KPS, age at transplantation and time from diagnosis to transplantation were protective factors ( $\mathrm{HR}=0.76,95 \% \mathrm{CI}$ : 0.64-0.90, $P=0.001 ; \mathrm{HR}=0.92$, $95 \%$ CI: $0.87-0.98, P=0.007$; and HR=0.98, 95\% CI: $0.97-$ $0.99, P<10^{-3}$, respectively) (Table 4 ).

No differences in NRM were noted between the three groups of patients in univariate analysis (Table 2, Figure 1D). Multivariate analysis demonstrated that patients' age

Table 2. Transplantation outcomes.

\begin{tabular}{|c|c|c|c|c|c|c|}
\hline & Haplo PTCy & UD 10/10 & UD $9 / 10$ & $P$ value & $\begin{array}{c}\text { P value } \\
\text { Haplo versus } \\
\text { UD 10/10 }\end{array}$ & $\begin{array}{c}\text { P value } \\
\text { Haplo versus } \\
\text { UD } 9 / 10\end{array}$ \\
\hline Leukemia-free survival & $22.8 \%(16.3-29.2)$ & $28 \%(25-30.9)$ & $22.2 \%(17.6-26.7)$ & NS & NS & NS \\
\hline Overall survival & $29.3 \%(22.1-36.6)$ & $34.7 \%(31.5-37.8)$ & $27.6 \%(22.7-32.5)$ & NS & NS & NS \\
\hline Relapse incidence & $52 \%(44.3-59.1)$ & $46.3 \%(43.1-49.4)$ & $51.1 \%(45.7-56.3)$ & NS & NS & NS \\
\hline Non-relapse mortality & $25.3 \%(19.2-31.8)$ & $25.7 \%(23.1-28.5)$ & $26.7 \%(22.2-31.4)$ & NS & NS & NS \\
\hline GRFS & $16.3 \%(10.6-21.9)$ & $16.4 \%(14-18.8)$ & $16 \%(12.1-19.9)$ & NS & NS & NS \\
\hline
\end{tabular}

Data are presented as percentage with 95\% confidence intervals in brackets. GRFS: graft-versus-host disease-free, relapse-free survival; haplo: haploidentical; PTCy: post-transplant cyclophosphamide; NS: not significant; UD: unrelated donors.
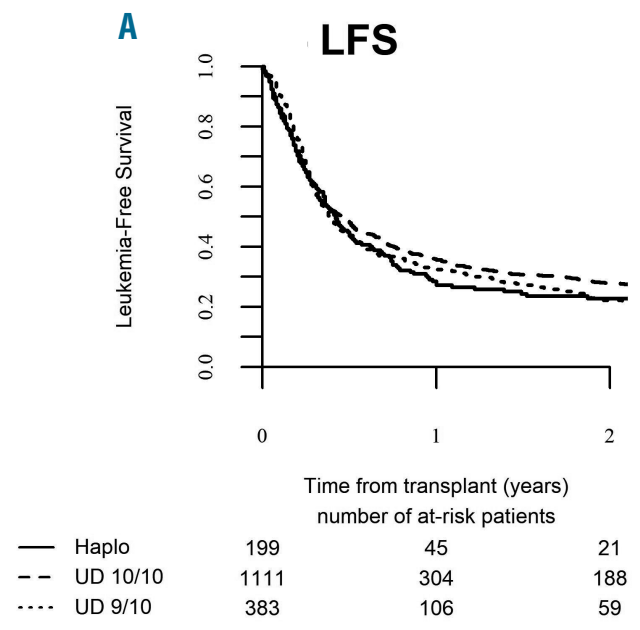

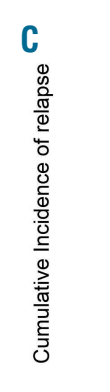

RI

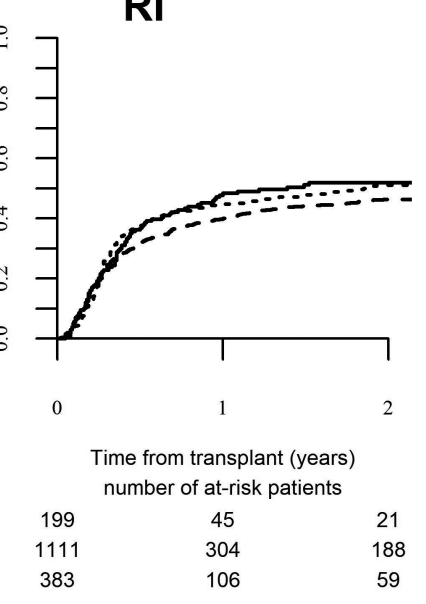

B

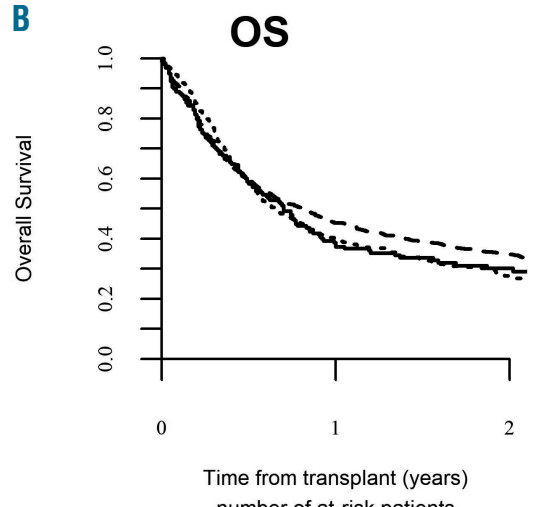

$\begin{array}{ll}\text { - } & \text { Haplo } \\ \ldots- & \text { UD } 10 / 10 \\ \ldots . & \text { UD } 9 / 10\end{array}$

\begin{tabular}{ccc}
\multicolumn{3}{c}{ number of at-risk patients } \\
199 & 60 & 26 \\
1111 & 384 & 234 \\
383 & 128 & 73
\end{tabular}

\section{NRM}

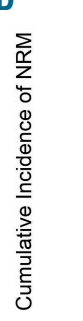

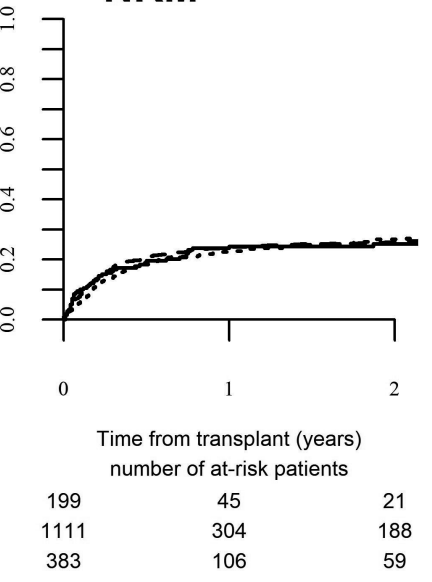

Figure 1. Leukemia-free survival, overall survival, relapse incidence and non-relapse mortality in patients with acute myeloid leukemia allografted during active disease. (A) The 2-year probability of leukemia-free survival (LFS) was $22.8 \%$ (95\% Cl: 16.3-29.2) in the group transplanted from a haploindentical donor with posttransplant cyclophosphamide (Haplo) versus 28\% (95\% Cl: 25-30.9) in the 10/10 HLA-matched unrelated donor group (UD 10/10), and 22.2\% (95\% Cl: 17.6-26.7) in the 9/10 HLA-mismatched unrelated donor group (UD 9/10) ( $P=N S$ ). (B) The 2-year probability of overall survival (OS) was 29.3\% (95\% Cl: 22.1-36.6) in the Haplo group versus $34.7 \%$ (95\% Cl: $31.5-37.8$ ) in the UD 10/10 and $27.6 \%$ (95\% Cl: $22.7-32.5)$ in the UD 9/10 groups (P=NS). (C) The 2 -year cumulative incidence of relapse (RI) was 52\% (95\% Cl: 44.3-59.1) in the Haplo group versus 46.3\% (95\% Cl: 43.1-49.4) in the UD 10/10 and 51.1\% (95\% Cl: 45.7-56.3) in the UD 9/10 groups ( $P=\mathrm{NS}$ ). (D) The 2-year cumulative incidence of non-relapse mortality (NRM) was $25.3 \%$ (95\% Cl: $19.2-31.8)$ in the Haplo group versus $25.7 \%$ (95\% Cl: 23.1 28.5 ) in the UD $10 / 10$ and $26.7 \%$ (95\% $\mathrm{Cl}: 22.2-31.4)$ in the UD $9 / 10$ groups ( $P=N S$ ). 
(per 10 years) and cytomegalovirus positivity were associated with higher NRM (HR=1.18, 95\% CI: 1.08-1.28, $P<10^{-3}$; and $\mathrm{HR}=1.37,95 \% \mathrm{CI}: 1.07-1.77, P=0.01$, respectively), while RIC compared to MAC and KPS $\geq 90$ were associated with lower NRM (HR=0.64, 95\% CI: 0.49-0.84, $P=0.001$; and $\mathrm{HR}=0.52,95 \% \mathrm{CI}: 0.41-0.64, P<10^{-3}$; respectively) (Table 4). Notably, no effect was observed for the type of donor.

In addition, no significant differences were found in GRFS according to donor type in the multivariate analysis. Three factors were associated with a better GRFS: longer time from diagnosis to transplantation, RIC versus MAC, and a KPS $\geq 90$. Patients with poor cytogenetics had a lower GRFS (Table 4).

As shown in Online Supplementary Table S2, most events happened within the first year after HSCT.

Finally, in order to reduce the effects of confounding factors, we performed a weighted analysis on propensity scores (weighted average treatment). The results of the weighted Kaplan-Meier and Cox analyses confirmed the study results as described in Table 5. In the weighted analysis on propensity scores, the Haplo PTCy group had a significantly lower incidence of grade III-IV acute GvHD compared to that of patients in the UD 10/10 group (8.9\% versus $14.5 \%$, respectively, $P=0.04)$, as confirmed by Cox analysis $(P=0.049)$.

\section{Causes of death}

Leukemia was the most common cause of death (accounting for $50 \%$ of the deaths in the Haplo PTCy group, $54 \%$ in the UD $10 / 10$ group, and $54.5 \%$ in the UD $9 / 10$ group). GvHD was the second most common, being the cause of death in $11.5 \%$ of the patients in the Haplo PTCy group, $12.1 \%$ in the UD 10/10 group, and $15.2 \%$ in the UD $9 / 10$ group. Infection was the cause of death in $27 \%, 20.8 \%$, and $20.6 \%$ of the patients in the Haplo PTCy, UD10/10 and UD 9/10 groups, respectively.

\section{Discussion}

In the present study, we compared the transplantation outcomes after Haplo HSCT with PTCy versus transplantation from matched (10/10) or mismatched (9/10) UD in AML patients with active disease. The LFS rate was about $25 \%$ and the OS rate about $30 \%$ after HSCT for this highrisk population with advanced disease with no difference between Haplo PTCy and UD 10/10 or 9/10. Rates of acute GvHD grades II-IV and of chronic GVHD were similar between Haplo PTCy and UD 10/10, and the same held true for the 2-year NRM. It is important to note that higher rates of grades II-IV acute GvHD and chronic GvHD were observed in the UD 9/10 group without there being an impact on RI. Although we could hypothesize a stronger graft-versus-leukemia effect after Haplo PTCy than after UD HSCT, we did not observe differences in terms of RI or LFS. This finding is in accordance with those of our previous study, in which we compared relapse rates between patients with primary refractory AML undergoing allogeneic transplantation from unrelated versus sibling donors and found no difference. ${ }^{13}$ One hypothesis is that, besides the very strong tolerance induction mediated by the PTCy in the Haplo setting in the case of active leukemia, the very aggressive biology of the disease and its refractoriness to several lines of chemotherapy lead to fast disease progression and relapse early after transplantaion. Thus, the graft-versus-leukemia effect, even across broad HLA disparities, is too weak or too slow to control the leukemia.

Of note, about $37 \%$ of patients received a RIC regimen in our study. As expected, the NRM rate was significantly lower in the RIC group than in the MAC group, with no difference in RI between the two groups. In all, LFS, OS, and GRFS were significantly better after RIC than after MAC. We could hypothesize that this is because even a high intensity conditioning regimen does not have a strong impact on this chemo-refractory leukemia. In our current study, 384 of the patients received a sequential approach with aplasia-inducing chemotherapy followed by the conditioning regimen. However, the question of which treatment should be used in a given patient cannot yet be answered. In a recent meta-analysis of leukemic patients with induction failure, Wattad et al. concluded that HSCT without prior salvage chemotherapy and HSCT in CR after salvage therapy resulted in comparable survival outcomes, and both strategies were significantly superior to HSCT performed after failure of salvage therapy. ${ }^{33}$ In the present study, no differences in outcome were found between patients who received MAC or sequential regimens. One hypothesis to explain this is that the refractoriness of the malignant leukemic clone to chemotherapy makes the conditioning regimen unable to induce remission, or even a transient response allowing sufficient time for the alloreactive cells to mediate the graft-versusleukemia effect. ${ }^{34}$

Importantly, an interval from diagnosis to transplant longer than the median was a negative prognostic factor for LFS, OS, RI and GRFS in multivariate analysis. These data, which are consistent with those of a study by Craddock et al. ${ }^{14}$ and our previous results, ${ }^{13}$ highlight the

Table 3. Univariate analysis for acute graft-versus-host disease and chronic graft-versus-host disease.

\begin{tabular}{lcccccc} 
& Haplo PTCy & UD 10/10 & UD 9/10 & P value & $\begin{array}{c}\text { P value } \\
\text { Haplo PICy } \\
\text { versus UD 10/10 }\end{array}$ & $\begin{array}{c}\text { P value } \\
\text { Haplo PICy } \\
\text { versus UD 9/10 }\end{array}$ \\
Acute GvHD II-IV & $28.2 \%(21.8-34.9)$ & $30.6 \%(27.8-33.4)$ & $36.3 \%(31.3-41.2)$ & 0.04 & NS & 0.03 \\
Acute GvHD III-IV & $8.9 \%(5.3-13.7)$ & $14 \%(11.9-16.2)$ & $16.1 \%(12.5-20.1)$ & NS & NS & 0.02 \\
\hline Chronic GvHD & $19.3 \%(13.6-25.7)$ & $25.6 \%(22.7-28.6)$ & $27.4 \%(22.6-32.4)$ & NS & NS & 0.04 \\
Extensive chronic GvHD & $11 \%(6.7-16.4)$ & $11.6 \%(9.6-13.9)$ & $11.6 \%(8.3-15.4)$ & NS & NS & NS \\
\hline PMN day 30 & $85.5 \%(79-90.2)$ & $92.3 \%(90.5-93.7)$ & $92.2 \%(88.9-94.6)$ & $<\mathbf{1 0}^{-3}$ & $<\mathbf{1 0}^{-3}$ & $<\mathbf{1 0}^{-3}$ \\
\hline
\end{tabular}

Data are presented as percentages with 95\% confidence intervals in brackets. ext: extensive; GvHD: graft-versus-host disease; haplo: haploidentical; PTCy: post-transplant; PMN: polymorphonuclear neutrophil; PTCy: post-transplant cyclophosphamide; UD: unrelated donors. 
Table 4. Multivariate analysis for leukemia-free survival, overall survival, relapse incidence, non-relapse mortality and graft-versus-host disease-free, relapse-free survival.

\begin{tabular}{lccc} 
& HR & CI & P value \\
$\begin{array}{l}\text { Leukemia-free survival } \\
\text { UD 10/10 versus Haplo }\end{array}$ & 0.97 & $0.75-1.24$ & NS \\
UD 9/10 versus Haplo & 1.05 & $0.80-1.37$ & NS \\
\hline Disease status: PRF-AML & 1 & & \\
$1^{\text {st }}$ relapse versus PRF-AML & 1.10 & $0.95-1.28$ & NS \\
$2^{\text {nd }}$ relapse versus PRF-AML & 1.31 & $1.03-1.65$ & $\mathbf{0 . 0 3}$ \\
Time from diagnosis to transplant & 0.99 & $0.98-0.99$ & $\mathbf{0 . 0 2}$ \\
\hline Age at transplant (per 10 years) & 1.00 & $0.95-1.05$ & NS \\
Year of transplant & 1.00 & $0.97-1.03$ & NS \\
\hline Conditioning regimen (ref=MAC) & 1 & & \\
RIC versus MAC & 0.84 & $0.71-0.99$ & $\mathbf{0 . 0 3}$ \\
Sequential strategy versus MAC & 0.97 & $0.8-1.17$ & NS \\
Poor cytogenetics & 1.35 & $1.11-1.62$ & $\mathbf{0 . 0 0 2}$ \\
\hline Karnofsky Performance Score $\geq 90$ & 0.66 & $0.58-0.76$ & $<\mathbf{1 0}{ }^{-3}$ \\
Stem cell source: PBS versus BM & 0.97 & $0.78-1.21$ & NS \\
\hline Previous autologous transplant & 0.95 & $0.68-1.33$ & NS \\
Female to male ratio & 0.89 & $0.74-1.06$ & NS \\
\hline Patient CMV positive & 1.22 & $1.05-1.41$ & 0.01 \\
Donor CMV positive & 0.99 & $0.87-1.14$ & NS \\
\hline Center (frailty) & & & $<\mathbf{1 0}^{-3}$ \\
\hline & & &
\end{tabular}

\section{Overall survival}

\begin{tabular}{lccc} 
UD 10/10 versus Haplo & 0.98 & $0.75-1.27$ & NS \\
UD 9/10 versus Haplo & 1.05 & $0.79-1.39$ & NS \\
\hline Disease status: PRF-AML & 1 & & \\
1 $^{\text {st }}$ relapse versus PRF-AML & 1.14 & $0.97-1.33$ & NS \\
$2^{\text {nd }}$ relapse versus PRF-AML & 1.35 & $1.05-1.72$ & $\mathbf{0 . 0 1 8}$ \\
Time from diagnosis to transplant & 0.99 & $0.99-1.00$ & $\mathbf{0 . 0 2}$ \\
\hline Age at transplant (per 10 years) & 1.04 & $0.99-1.10$ & NS \\
Year of transplant & 1.00 & $0.97-1.03$ & NS \\
\hline Conditioning regimen (ref=MAC) & 1 & & \\
RIC versus MAC & 0.74 & $0.62-0.88$ & $<\mathbf{1 0}$ \\
Sequential strategy versus MAC & 0.91 & $0.75-1.12$ & NS \\
Poor cytogenetics & 1.28 & $1.06-1.56$ & $\mathbf{0 . 0 1}$ \\
\hline Karnofsky Performance Score $\geq 90$ & 0.62 & $0.54-0.71$ & $<\mathbf{1 0}$ \\
Stem cell source: PBS versus BM & 0.97 & $0.77-1.22$ & NS \\
\hline Previous autologous transplant & 0.94 & $0.66-1.35$ & NS \\
Female to male ratio & 0.90 & $0.75-1.09$ & NS \\
\hline Patient CMV positive & 1.27 & $1.09-1.49$ & $\mathbf{0 . 0 0 2}$ \\
Donor CMV positive & 0.96 & $0.83-1.11$ & NS \\
\hline Center (frailty) & & & $<\mathbf{1 0}{ }^{-3}$ \\
\hline
\end{tabular}

\section{Relapse incidence}

\begin{tabular}{lccc} 
UD 10/10 versus Haplo & 0.92 & $0.67-1.25$ & NS \\
UD 9/10 versus Haplo & 1.03 & $0.74-1.47$ & NS \\
\hline Disease status: PRF-AML & 1 & & \\
$1^{\text {st }}$ relapse versus PRF-AML & 1.32 & $1.09-1.60$ & $\mathbf{0 . 0 0 5}$ \\
$2^{\text {nd }}$ relapse versus PRF-AML & 1.64 & $1.21-2.21$ & $\mathbf{0 . 0 0 1}$ \\
Time from diagnosis to transplant & 0.98 & $0.97-0.99$ & $<\mathbf{1 0}^{-3}$ \\
\hline Age at transplant (per 10 years) & 0.92 & $0.87-0.98$ & $\mathbf{0 . 0 0 7}$ \\
Year of transplant & 0.98 & $0.94-1.01$ & NS \\
\hline Conditioning regimen (ref=MAC) & 1 & \multicolumn{2}{|}{} \\
RIC versus MAC & 0.97 & $0.79-1.19$ & NS \\
& & \multicolumn{2}{c}{ continued in the next coloum }
\end{tabular}

continued from the previous coloum

\begin{tabular}{lccc} 
& HR & Cl & P value \\
Sequential strategy versus MAC & 1.07 & $0.85-1.36$ & NS \\
Poor cytogenetics & 1.48 & $1.18-1.85$ & $<\mathbf{1 0}^{-3}$ \\
\hline Karnofsky Performance Score $\geq 90$ & 0.76 & $0.64-0.90$ & $\mathbf{0 . 0 0 1}$ \\
Stem cell source: PBS versus BM & 0.90 & $0.69-1.18$ & NS \\
\hline Previous autologous transplant & 0.99 & $0.65-1.54$ & NS \\
Female to male ratio & 0.89 & $0.71-1.12$ & NS \\
\hline Patient CMV positive & 1.15 & $0.96-1.38$ & NS \\
Donor CMV positive & 0.97 & $0.82-1.15$ & NS \\
\hline Center (frailty) & & & $<\mathbf{1 0}^{-3}$
\end{tabular}

\section{Non-relapse mortality}

\begin{tabular}{lccc} 
UD 10/10 versus Haplo & 1.01 & $0.67-1.52$ & NS \\
UD 9/10 versus Haplo & 1.03 & $0.66-1.61$ & NS \\
\hline Disease status: PRF-AML & 1 & & \\
1 $^{\text {st }}$ relapse versus PRF-AML & 0.88 & $0.70-1.12$ & NS \\
$2^{\text {nd }}$ relapse versus PRF-AML & 1.03 & $0.71-1.50$ & NS \\
Time from diagnosis to transplant & 1.00 & $0.99-1.01$ & NS \\
\hline Age at transplant (per 10 years) & 1.18 & $1.08-1.28$ & $<\mathbf{1 0}^{-3}$ \\
Year of transplant & 1.04 & $0.99-1.09$ & NS \\
\hline Conditioning regimen (ref=MAC) & 1 & & \\
RIC versus MAC & 0.64 & $0.49-0.84$ & $\mathbf{0 . 0 0 1}$ \\
Sequential strategy versus MAC & 0.80 & $0.59-1.09$ & NS \\
Poor cytogenetics & 1.10 & $0.79-1.55$ & NS \\
\hline Karnofsky Performance Score $\geq 90$ & 0.52 & $0.41-0.64$ & $<\mathbf{1 0}$ \\
Stem cell source: PBS versus BM & 1.19 & $0.81-1.75$ & NS \\
\hline Previous autologous transplant & 0.99 & $0.57-1.72$ & NS \\
Female to male & 0.87 & $0.63-1.18$ & NS \\
\hline Patient CMV positive & 1.37 & $1.07-1.77$ & $\mathbf{0 . 0 1}$ \\
Donor CMV positive & 1.03 & $0.83-1.29$ & NS \\
\hline Center (frailty) & & & NS
\end{tabular}

GRFS

\begin{tabular}{lccc} 
UD 10/10 versus Haplo & 1.09 & $0.87-1.39$ & NS \\
UD 9/10 versus Haplo & 1.11 & $0.86-1.43$ & NS \\
\hline Disease status: PRF-AML & 1 & & \\
1 $^{\text {st }}$ relapse versus PRF-AML & 1.05 & $0.91-1.21$ & NS \\
$2^{\text {nd }}$ relapse versus PRF-AML & 1.22 & $0.98-1.52$ & NS \\
Time from diagnosis to transplant & 0.99 & $0.98-0.99$ & $\mathbf{0 . 0 2}$ \\
\hline Age at transplant (per 10 years) & 0.98 & $0.93-1.02$ & NS \\
Year of transplant & 1.02 & $0.99-1.05$ & NS \\
\hline Conditioning regimen (ref=MAC) & 1 & & \\
RIC versus MAC & 0.86 & $0.74-0.99$ & $\mathbf{0 . 0 4 7}$ \\
Sequential strategy versus MAC & 0.93 & $0.78-1.11$ & NS \\
Poor cytogenetics & 1.20 & $1.00-1.44$ & $\mathbf{0 . 0 4 6}$ \\
\hline Karnofsky Performance Score $\geq 90$ & 0.68 & $0.60-0.77$ & $<\mathbf{1 0}$ \\
Stem cell source: PB versus BM & 1.15 & $0.94-1.42$ & NS \\
\hline Previous autologous transplant & 0.95 & $0.69-1.31$ & NS \\
Female to male ratio & 0.85 & $0.71-1.01$ & NS \\
\hline Patient CMV positive & 1.14 & $0.99-1.31$ & NS \\
Donor CMV positive & 0.98 & $0.86-1.12$ & NS \\
\hline Center (frailty) & & & $\mathbf{0 . 0 1}$
\end{tabular}

AML: acute myeloid leukemia; BM: bone marrow; CI: confidence interval; CMV cytomegalovirus; haplo: haploidentical; HR: hazard ratio; MAC: myeloablative conditioning; NS: not significant; PB: peripheral blood PRF-AML; PRF: primary refractory acute myeloid leukemia; Rel: relapse; RIC: reduced intensity conditioning; UD: unrelated donor. 
Table 5. Weighted Cox model for leukemia-free survival, overall survival, relapse incidence, non-relapse mortality, graft-versus-host disease-free, relapse-free survival and graft-versus-host disease.

\begin{tabular}{lccccc} 
& Haplo PICy & UD 10/10 & UD 9/10 & $\begin{array}{c}\text { P value } \\
\text { Haplo PTCy } \\
\text { versus UD 10/10 }\end{array}$ & $\begin{array}{c}\text { P value } \\
\text { Haplo PICy } \\
\text { versus UD 9/10 }\end{array}$ \\
Leukemia-free survival & $22.8 \%(17.1-30.2)$ & $25.6 \%(19.4-33.7)$ & $21.6 \%(15-30.9)$ & NS & NS \\
Overall survival & $29.3 \%(22.9-37.5)$ & $32.2 \%(25.4-40.8)$ & $25.4 \%(18.3-35.4)$ & NS & NS \\
\hline Relapse incidence & $52 \%(43.9-58.8)$ & $48.2 \%(43.6-52.5)$ & $53.2 \%(45.6-59.7)$ & NS & NS \\
Non-relapse mortality & $25.3 \%(18.7-31.3)$ & $26.2 \%(22.3-29.9)$ & $25.2 \%(19.3-30.6)$ & NS & NS \\
\hline GRFS & $16.2 \%(11.5-22.9)$ & $17.1 \%(11.9-24.4)$ & $16.1 \%(10.5-24.7)$ & NS & NS \\
Acute GvHD II-IV & $28.2 \%(21.3-34.5)$ & $31.3 \%(27-35.3)$ & $40.4 \%(32.8-47.2)$ & NS & 0.01 \\
\hline Acute GvHD III-IV & $8.9 \%(4.6-13)$ & $14.5 \%(11.4-17.4)$ & $20.1 \%(13.5-26.2)$ & 0.04 & 0.005 \\
Chronic GvHD & $19.3 \%(13-25.1)$ & $22.9 \%(19.1-26.6)$ & $25.5 \%(19.1-31.4)$ & NS & 0.04 \\
\hline Extensive chronic GvHD & $11.3 \%(6.2-16.1)$ & $11.2 \%(8.5-13.8)$ & $11.1 \%(6.6-15.3)$ & NS & NS \\
\hline
\end{tabular}

Data are presented as percentages with 95\% confidence intervals in brackets; GvHD: graft-versus-host disease; GRFS: graft-versus-host disease-free, relapse-free survival; Haplo: haploidentical; not significant; PTCy: post-transplant cyclophosphamide; UD: unrelated donors.

urgent need to search for a donor for AML patients with active disease who lack a matched sibling donor. Haploidentical donors are available for the majority of patients, providing access to further stem cell donations or donor lymphocyte infusions as needed. ${ }^{35}$ Furthermore, it is a factor on which the physicians can have an influence, unlike many other factors. In accordance, Wattad et al. showed that patients transplanted during refractory disease after salvage therapy had a significantly poorer outcome compared to that of patients who proceeded directly to transplantation, and those transplanted in CR after salvage therapy. ${ }^{12}$ Thus, we could recommend not to try additional lines of chemotherapy to achieve CR in patients with active disease, but to take advantage of the first available donor in order to proceed to transplantation.

The cytogenetic characterization of the leukemia represents a major prognostic factor for LFS, RI, OS, and GRFS. ${ }^{36,37}$ We previously reported that poor-risk cytogenetics was an adverse pre-HSCT variable in patients with primary refractory AML who underwent HSCT with a graft from a matched sibling or matched UD. ${ }^{13}$ Accordingly, in the present study, primary refractory AML with poor cytogenetic characteristics was associated, at 2 years, with a significant decrease in LFS and OS, and increase in RI. These data pave the road for investigating additional approaches relying on sequential conditioning regimens and/or post-transplant treatments. ${ }^{38-42}$

Being retrospective and registry-based, this study has some limitations: several of the patients' characteristics differed between the groups. We addressed this limitation, at least in part, by using the propensity score technique. In addition, there was a relative inherent selection process for HSCT in our study and a relative lack of infor- mation on the reasons for an EBMT center allocating patients to HSCT from a haploidentical donor versus UD, so that distinguishing the choice of the donor from the role of a potential center effect is difficult. Finally, the counts of circulating and bone marrow blasts at the time of HSCT were missing for a substantial number of patients. However, the aim of this analysis was to compare the two types of donors using EBMT registry data. The design of the study and inclusion criteria were intended to answer this clinical question and were not, therefore, adapted for developing a prognostic score. There are ongoing trials aiming to compare outcomes after Haplo PTCy versus UD in hematologic malignancies, but they do not focus specifically on the setting of active AML disease (NCT02623309). Therefore, in the absence of any prospect of such comparative studies, our data suggest that haploidentical donors are equally effective as 10/10 matched and 9/10 mismatched UD for allogeneic transplantation in patients with active AML.

In conclusion, our results indicate that, when an HLAidentical sibling donor is not available for an AML patient with active disease who is, otherwise, a candidate for HSCT, a haploidentical donor may be used with the expectation of similar rates of NRM, LFS, OS, and GRFS at 2 years, compared with those achieved with 10/10 matched and 9/10 mismatched UD.

\section{Acknowledgments}

We thank all the European Society for Blood and Marrow Transplantation (EBMT) participating centers and national registries for providing patients' data for the study, and data managers for their valuable contribution (Online Supplementary Data). Further information is available at the EBMT web site.

\section{References}

1. Dohner H, Estey E, Grimwade D, et al. Diagnosis and management of AML in adults: 2017 ELN recommendations from an international expert panel. Blood. 2017;129 (4):424-447.
2. Thol F, Schlenk RF, Heuser M, Ganser A. How I treat refractory and early relapsed acute myeloid leukemia. Blood. 2015;126 (3):319-327.

3. Schlenk RF, Frech P, Weber D, et al. Impact of pretreatment characteristics and salvage strategy on outcome in patients with relapsed acute myeloid leukemia. Leukemia. 2017;31(5):1217-1220.
4. Dombret H, Gardin C. An update of current treatments for adult acute myeloid leukemia. Blood. 2016;127(1):53-61.

5. Revesz D, Chelghoum Y, Le OH, Elhamri M, Michallet M, Thomas X. Salvage by timed sequential chemotherapy in primary resistant acute myeloid leukemia: analysis of prognostic factors. Ann Hematol. 2003;82 (11):684-690 
6. Duval M, Klein JP, He W, et al. Hematopoietic stem-cell transplantation for acute leukemia in relapse or primary induction failure. J Clin Oncol. 2010;28(23):37303738.

7. Nagler A, Savani BN, Labopin M, et al. Outcomes after use of two standard ablative regimens in patients with refractory acute myeloid leukaemia: a retrospective, multicentre, registry analysis. Lancet Haematol. 2015;2(9):e384-392.

8. Gyurkocza B, Lazarus HM, Giralt S. Allogeneic hematopoietic cell transplantation in patients with AML not achieving remission: potentially curative therapy. Bone Marrow Transplant. 2017:52(8):1083-1090.

9. Dohner H, Weisdorf DJ, Bloomfield CD. acute myeloid leukemia. N Engl J Med. 2015;373(12):1136-1152.

10. Fung HC, Stein A, Slovak M, et al. A longterm follow-up report on allogeneic stem cell transplantation for patients with primary refractory acute myelogenous leukemia: impact of cytogenetic characteristics on transplantation outcome. Biol Blood Marrow Transplant. 2003;9(12):766-771.

11. Michallet M, Thomas X, Vernant JP, et al. Long-term outcome after allogeneic hematopoietic stem cell transplantation for advanced stage acute myeloblastic leukemia: a retrospective study of 379 patients reported to the Societe Francaise de Greffe de Moelle (SFGM). Bone Marrow Transplant. 2000;26(11):1157-1163.

12. Wattad M, Weber D, Dohner K, et al. Impact of salvage regimens on response and overall survival in acute myeloid leukemia with induction failure. Leukemia. 2017;31(6):1306-1313.

13. Brissot E, Labopin M, Stellies $M$, et al. Comparison of matched sibling donors versus unrelated donors in allogeneic stem cell transplantation for primary refractory acute myeloid leukemia: a study on behalf of the Acute Leukemia Working Party of the EBMT. J Hematol Oncol. 2017;10(1):130.

14. Craddock C, Labopin M, Pillai S, et al. Factors predicting outcome after unrelated donor stem cell transplantation in primary refractory acute myeloid leukaemia. Leukemia. 2011;25(5):808-813.

15. Jabbour E, Daver N, Champlin R, et al. Allogeneic stem cell transplantation as initial salvage for patients with acute myeloid leukemia refractory to high-dose cytarabinebased induction chemotherapy. Am J Hematol. 2014;89(4):395-398.

16. Lee CJ, Savani BN, Mohty M, et al. Haploidentical hematopoietic cell transplantation for adult acute myeloid leukemia: a position statement from the Acute Leukemia Working Party of the European Society for Blood and Marrow Transplantation. Haematologica. 2017;102 (11):1810-1822

17. Passweg JR, Baldomero H, Bader P, et al. Use of haploidentical stem cell transplantation continues to increase: the 2015 European Society for Blood and Marrow Transplant activity survey report. Bone Marrow Transplant. 2017;52(6):811-817.

18. Bashey A, Zhang X, Sizemore CA, et al. Tcell-replete HLA-haploidentical hematopoietic transplantation for hematologic malignancies using post-transplantation cyclophosphamide results in outcomes equivalent to those of contemporaneous HLA-matched related and unrelated donor transplantation. J Clin Oncol. 2013;31(10): 1310-1316.

19. Ciurea SO, Zhang MJ, Bacigalupo AA, et al. Haploidentical transplant with posttransplant cyclophosphamide vs matched unrelated donor transplant for acute myeloid leukemia. Blood. 2015;126(8):1033-1040.

20. Bashey A, Zhang X, Jackson K, et al. Comparison of outcomes of hematopoietic cell transplants from T-replete haploidentical donors using post-transplantation cyclophosphamide with 10 of 10 HLA-A, -B, -C, -DRB1, and -DOB1 allele-matched unrelated donors and HLA-identical sibling donors: a multivariable analysis including disease risk index. Biol Blood Marrow Transplant. 2016;22(1):125-133.

21. Rashidi A, DiPersio JF, Westervelt P, et al. Comparison of outcomes after peripheral blood haploidentical versus matched unrelated donor allogeneic hematopoietic cell transplantation in patients with acute myeloid leukemia: a retrospective singlecenter review. Biol Blood Marrow Transplant. 2016;22(9):1696-1701

22. Piemontese S, Ciceri F, Labopin M, et al. A comparison between allogeneic stem cell transplantation from unmanipulated haploidentical and unrelated donors in acute leukemia. J Hematol Oncol. 2017;10(1):24.

23. Versluis J, In 't Hout FE, Devillier R, et al. Comparative value of post-remission treatment in cytogenetically normal AML subclassified by NPM1 and FLT3-ITD allelic ratio. Leukemia. 2017;31(1):26-33.

24. Grimwade D, Hills RK, Moorman AV, et al. Refinement of cytogenetic classification in acute myeloid leukemia: determination of prognostic significance of rare recurring chromosomal abnormalities among 5876 younger adult patients treated in the United Kingdom Medical Research Council trials. Blood. 2010;116(3):354-365.

25. Bacigalupo A, Ballen K, Rizzo D, et al. Defining the intensity of conditioning regimens: working definitions. Biol Blood Marrow Transplant. 2009;15(12):1628-1633.

26. Schmid C, Schleuning M, Ledderose G, Tischer J, Kolb HI. Sequential regimen of chemotherapy, reduced-intensity conditioning for allogeneic stem-cell transplantation, and prophylactic donor lymphocyte transfusion in high-risk acute myeloid leukemia and myelodysplastic syndrome. J Clin Oncol. 2005·23(24):5675-5687.

27. Malard F, Labopin M, Stuhler G, et al. Sequential intensified conditioning Regimen allogeneic hematopoietic stem cell transplantation in adult patients with intermediate- or high-risk acute myeloid leukemia in complete remission: a study from the Acute Leukemia Working Party of the European Group for Blood and Marrow Transplantation. Biol Blood Marrow Transplant. 2017;23(2):278-284.

28. Ringden $O$, Labopin M, Schmid C, et al. Sequential chemotherapy followed by reduced-intensity conditioning and allogeneic haematopoietic stem cell transplantation in adult patients with relapse or refractory acute myeloid leukaemia: a survey from the Acute Leukaemia Working Party of
EBMT. Br J Haematol. 2017;176(3):431-439.

29. Glucksberg H, Storb R, Fefer A, et al. Clinical manifestations of graft-versus-host disease in human recipients of marrow from HL-Amatched sibling donors. Transplantation. 1974;18(4):295-304.

30. Terwey TH, Le Duc TM, Hemmati PG, et al $\mathrm{NIH}$-defined graft-versus-host disease and evidence for a potent graft-versus-leukemia effect in patients with acute lymphoblastic leukemia. Ann Oncol. 2013;24(5):1363-1370.

31. Ruggeri A, Labopin M, Ciceri F, Mohty M, Nagler A. Definition of GvHD-free, relapsefree survival for registry-based studies: an ALWP-EBMT analysis on patients with AML in remission. Bone Marrow Transplant. 2016;51(4):610-611.

32. Clark TG, Altman DG, De Stavola BL. Quantification of the completeness of follow-up. Lancet. 2002;359(9314):1309-1310.

33. Wattad M, Weber D, Dohner K, et al. Impact of salvage regimens on response and overall survival in acute myeloid leukemia with induction failure. Leukemia. 2017;31 (6):1306-1313

34. Raanani P, Dazzi F, Sohal J, et al. The rate and kinetics of molecular response to donor leucocyte transfusions in chronic myeloid leukaemia patients treated for relapse after allogeneic bone marrow transplantation. $\mathrm{Br}$ Haematol. 1997;99(4):945-950.

35. Kanakry CG, Fuchs EJ, Luznik L. Modern approaches to HLA-haploidentical blood or marrow transplantation. Nat Rev Clin Oncol. 2016;13(2):132.

36. Byrd JC, Mrozek K, Dodge RK, et al. Pretreatment cytogenetic abnormalities are predictive of induction success, cumulative incidence of relapse, and overall survival in adult patients with de novo acute myeloid leukemia: results from Cancer and Leukemia Group B (CALGB 8461). Blood. 2002;100(13):4325-4336.

37. Grimwade D, Hills RK, Moorman AV, et al Refinement of cytogenetic classification in acute myeloid leukemia: determination of prognostic significance of rare recurring chromosomal abnormalities among 5876 younger adult patients treated in the United Kingdom Medical Research Council trials. Blood. 2010;116(3):354-365

38. Tsirigotis P, Byrne M, Schmid C, et al Relapse of AML after hematopoietic stem cell transplantation: methods of monitoring and preventive strategies. A review from the ALWP of the EBMT. Bone Marrow Transplant. 2016;51(11):1431-1438.

39. Stein EM, DiNardo CD, Pollyea DA, et al Enasidenib in mutant IDH2 relapsed or refractory acute myeloid leukemia. Blood. 2017;130(6):722-731.

40. Kayser S, Levis MJ, Schlenk RF. Midostaurin treatment in FLT3-mutated acute myeloid leukemia and systemic mastocytosis. Expert Rev Clin Pharmacol. 2017;10(11):1177-1189.

41. Antar A, Otrock ZK, El-Cheikh J, et al. Inhibition of FLT3 in AML: a focus on sorafenib. Bone Marrow Transplant. 2017;52 (3):344-351.

42. Konopleva M, Pollyea DA, Potluri J, et al Efficacy and biological correlates of response in a phase II study of venetoclax monotherapy in patients with acute myelogenous leukemia. Cancer Discov. 2016;6(10):11061117. 